\title{
AN ALTERNATIVE NON-INVASIVE TREATMENT FOR PEYRONIE'S DISEASE
}

\author{
JOAQUIM A. CLARO, CARLO C. PASSEROTTI, ANTONIO C. FIGUEIREDO NETO, \\ ARCHIMEDES NARDOZZA JR., VALDEMAR ORTIZ, MIGUEL SROUGI
}

\author{
Division of Urology, Paulista School of Medicine, Federal University of São Paulo, UNIFESP, São Paulo, \\ SP, Brazil
}

\begin{abstract}
Objective: Surgical correction of the deformity and plaque caused by Peyronie's disease has some important disadvantages and extracorporeal shockwave therapy (ESWT) emerged as a new promising therapy. We evaluated prospectively the efficacy and safety of the association of high dose vitamin $\mathrm{E}$ and ESWT as a non-invasive treatment for the disease.

Materials and Methods: Twenty-five patients 42 to 68 years old $($ mean $=54)$ presenting penile deviation and sexual distress caused by Peyronie's disease were treated in a non-invasive manner. The time of penile deviation ranged from 16 to 52 months $($ mean $=30)$. All patients had previous unsuccessful treatment for Peyronie's disease. The angulation's deformity of the penis was assessed by photography at home. The patients received vitamin $\mathrm{E}(1.200 \mathrm{mg}$ daily) during 3 months and underwent 3 to 6 sessions (mean $=3$ ) of ESWT (3,000 to 4,000 shockwaves) at a power level of 1 to 2 at 1 -week intervals.

Results: From 25 patients treated, 16 (64\%) reported an improvement in penile angulation, with a mean reduction of 21 degrees (10 to 40). Eight patients reported improvement in their spontaneous erections. Overall, the patients presented only minimal bruising at the site of treatment and skin hematoma. Four patients presented urethral bleeding. The mean angulation after treatment in the control group was 48.67 degrees (30 - 70) and in the study group was 24.42 degrees $(0-70)$, statistically significant.

Conclusion: Considering the common complications and the unsatisfactory outcome of the surgical correction for Peyronie's disease, the association of high dose vitamin E and ESWT represents a good option for a non-invasive, effective and safe treatment of the penile deformity.
\end{abstract}

Key words: penis; Peyronie's disease; extracorporeal shockwave lithotripsy; vitamin E; treatment. Int Braz J Urol. 2004; 30: 199-204

\section{INTRODUCTION}

Peyronie's disease refers to acquired penile deformities during erection (curvature, indentation, hourglass or shortening). Peyronie's disease is characterized by fibrotic plaques of the tunica albuginea of the penis (1). Over 250 years after its first description, the etiology of the Peyronie's lesion remains unclear. The most accepted hypothesis is that the Peyronie's plaques are caused by aberrant wound healing and scar formation, due to consecutive trauma during intercourse (2-4). Penile deviation is one result of the inelastic plaque, limiting extensibility of the penile shaft and causing an angled erection (5). The symptomatic incidence of Peyronie's disease has been estimated at $1 \%$. In white men, the average age 
at onset of Peyronie's disease is 53 years. The asymptomatic prevalence is estimated at $0.4 \%$ to $1.0 \%$. In another study with 100 men without known Peyronie's disease, $22 \%$ on autopsy were found to have fibrotic lesions of the tunica albuginea compatible with Peyronie's disease (6-9).

Peyronie's disease may cause significant distress, affecting the quality of life of both the patient and his partner. Psychological effects associated with the disease were reported by $77 \%$ of patients (7). Besides, there is a clearly suggestion that the clinical incidence of Peyronie's disease is increasing (10). Probably, this increase is associated and seems to coincide with the use of erection-enhancing medications. Although Peyronie's disease is characterized as a gradual spontaneous resolution process, certain features are associated with a lack of spontaneous resolution, including greater than 2-year duration. The treatment may be clinical or surgical. Oral systemic agents include potassium p-amino benzoate, tamoxifen, acetyl-L-carnitine, colchicines and vitamin E (12). On the other hand, there are quite a lot of surgical procedures to repair penile deformities; all of them are invasive, with significant disadvantages to the patient. Recently, encouraging results with the extra corporeal shockwave therapy (ESWT) were reported $(12,13)$.

We prospectively evaluated the association of high dose vitamin E and ESWT in the treatment of the penile deviation caused by Peyronie's disease.

\section{MATERIALS AND METHODS}

We studied 40 patients with sexual distress due to penile deviation caused by Peyronie's disease. Patients' age ranged from 42 to 68 years $($ mean $=54)$ and the time of penile deviation ranged from 16 to 52 months (mean $=30$ ).

Penile pain was referred by 35 patients but it was not the reason for treatment in any case. Eleven patients presented mild hypertension, 8 had diabete's and 3 have had a radical retropubic prostatectomy. Fifteen patients presented erectile dysfunction, 7 were treated with $50 \mathrm{mg}$ of sildenafil, 4 with $20 \mathrm{mg}$ tadalafil, 3 with $10 \mathrm{mg}$ vardenafil, and 1 with intracavernous injection therapy using tri-mix. All of the 40 patients were previously treated for the penile deviation with- out success. The previous therapies were low dose of vitamin E, tamoxifen, colchicines, potassium p-amino benzoate and intralesional injections of steroids and verapamil. All patients refused any kind of surgery.

The angulation of the penis was assessed by photography during a normal erection at home, and the diagnosis was confirmed by palpation of the plaque. The size and site of the plaques were assessed by ultrasonography.

The patients were divided into 2 groups: Group - 1, composed of 15 patients that were treated only by high dose vitamin E (1.200 mg daily) during 3 months, and Group - 2, composed of 25 patients that received vitamin $\mathrm{E}$ and were submitted to ESWT. The patients underwent 3 to 6 (median $=3$ ) sessions of ESWT at level-I of intensity, using the Lithostar Plus machine (Siemens, Germany), with 1-week interval. The accurate delivery of the shockwaves to the plaques was monitored by a real-time in line ultrasound scanner aided by palpation. Treatment results were assessed by the patient, subjectively. Besides, the objective evaluation was performed by photographs taken at home, palpation of the plaque and ultrasonographic measurements.

The results were shown as means and standard deviations. Two tailed " $t$ " test was used with Bonferroni correction for statistical comparison. $\mathrm{P}$ value $<0.01$ was considered statistically significant. Trying to predict association between the objective data and subjective response variable, we used logistic regression.

\section{RESULTS}

The treatment was very well tolerated, the high dosage of vitamin $\mathrm{E}$ had no side effects and the ESWT caused no pain. The use of analgesics was not necessary. The shock waves therapy presented only minor complications including penile bruising and surface bleeding at the point of entry of the shock waves in 19 patients. Two patients developed urethral bleeding, that settled with conservative treatment. The follow-up ranged from 5 to 13 months (mean $=8.5)$. There is no statistical difference between groups regarding age, diameter $(p=0.089)$ and angulation $(\mathrm{p}=0.25)$. 
Of the 25 patients treated, $18(72 \%)$ reported a significant decrease on the angulation deformity of the penis (Figure-1). Five patients reported discrete improvement in the disease and 2 reported no improvement in penile angulation. Eight patients referred improvement in their spontaneous erections. Eleven patients reported that subjectively the plaque had diminished. On palpation, the plaques were not detected in 2 patients and had diminished in other 11 patients.

The ultrasound did not detect five plaques that remained easily palpable even by the patients. On the other hand, in seven patients with subjective and palpation improvements the ultrasonographic findings did not change. One patient, otherwise, reported his
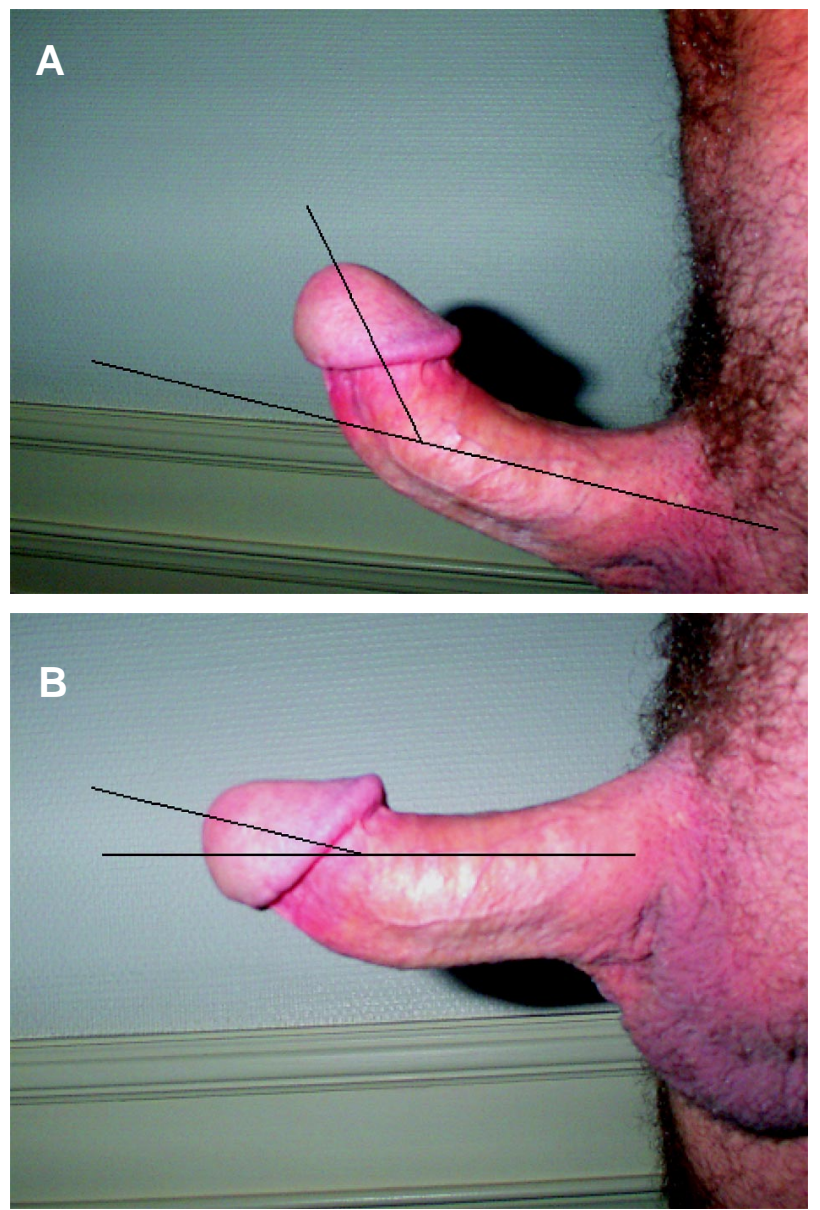

Figure 1 - A) Penile deviation due to Peyronie's disease. Before extracorporeal shockwave therapy. B) Penile shaft after 3 extracorporeal shockwave therapy sessions.
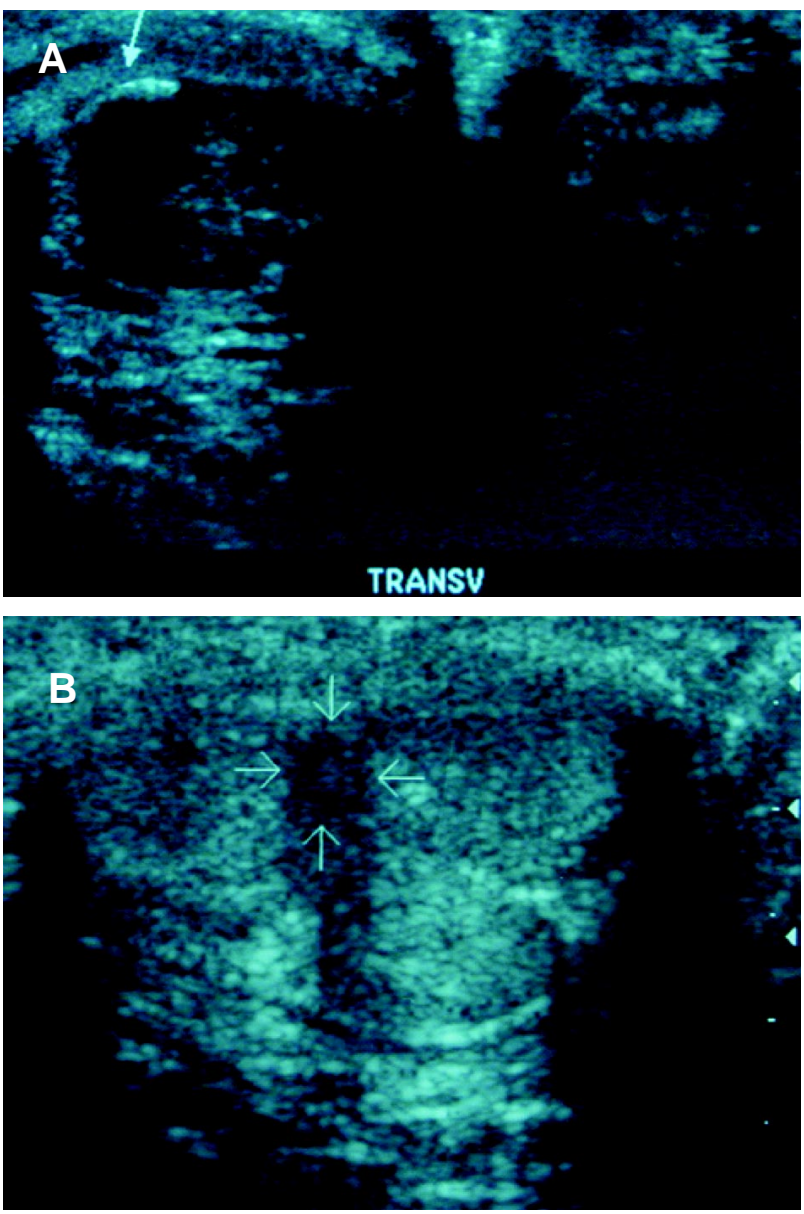

Figure 2 - A) Ultrasound showing Peyronie's plaque. Before extracorporeal shockwave therapy. B) Ultrasound after 3 extracorporeal shockwave therapy sessions.

penis was totally straight and had no detectable plaque either on palpation or on ultrasound (Figure-2).

The mean diameters and the mean angulations are shown on Tables 1 and 2.

When compared the greater diameter and the angulation preceding the procedure and after ESWT there were an important difference in diameter $(\mathrm{p}=$ $0.0001)$ and angulation ( $\mathrm{p}=0.0001)$, attesting the good response of ESWT.

Figures 3 and 4 show the diameter and angle before and after treatment.

The binary logistic regression showed that age $(\mathrm{p}=0.997, \mathrm{OR}=1)$, diameter $(\mathrm{p}=0.31, \mathrm{OR}=$ $2.2)$ and angulation $(\mathrm{p}=0.697, \mathrm{OR}=0.98)$ could not predict good or poor response. 


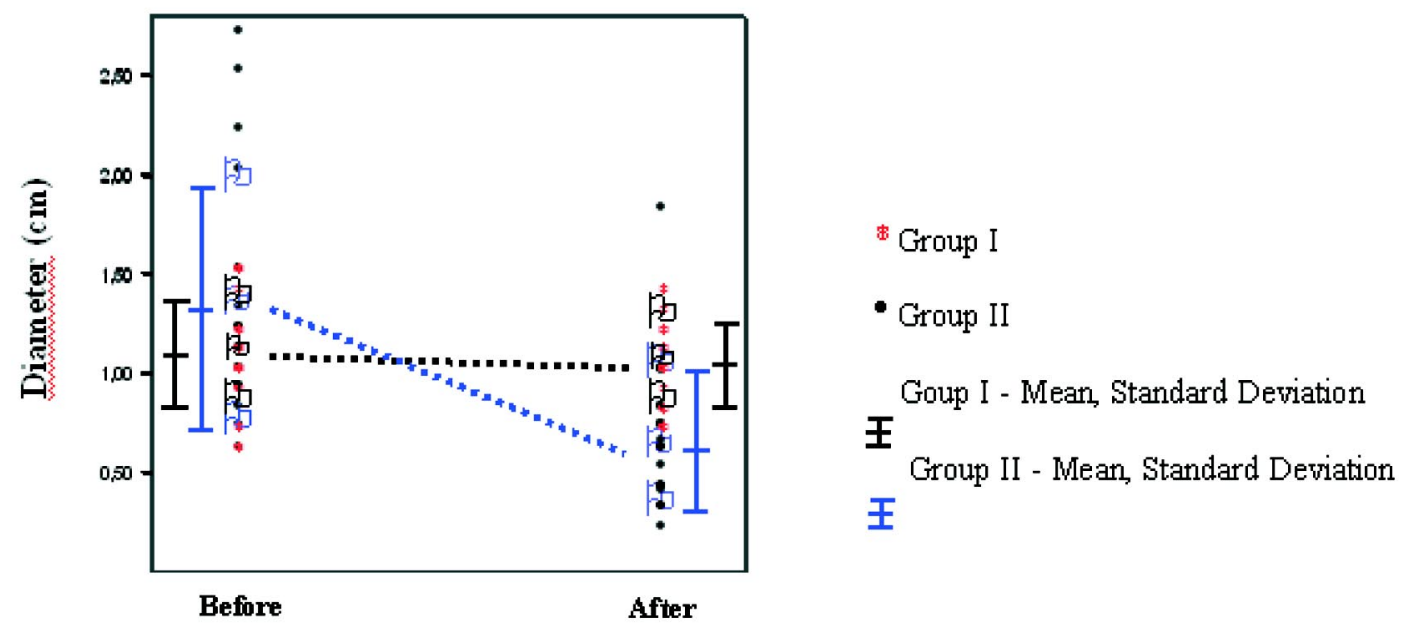

Figure 3 - Plaque diameter (cm) of patients from control (I) and study (II) groups (mean and standard deviation).

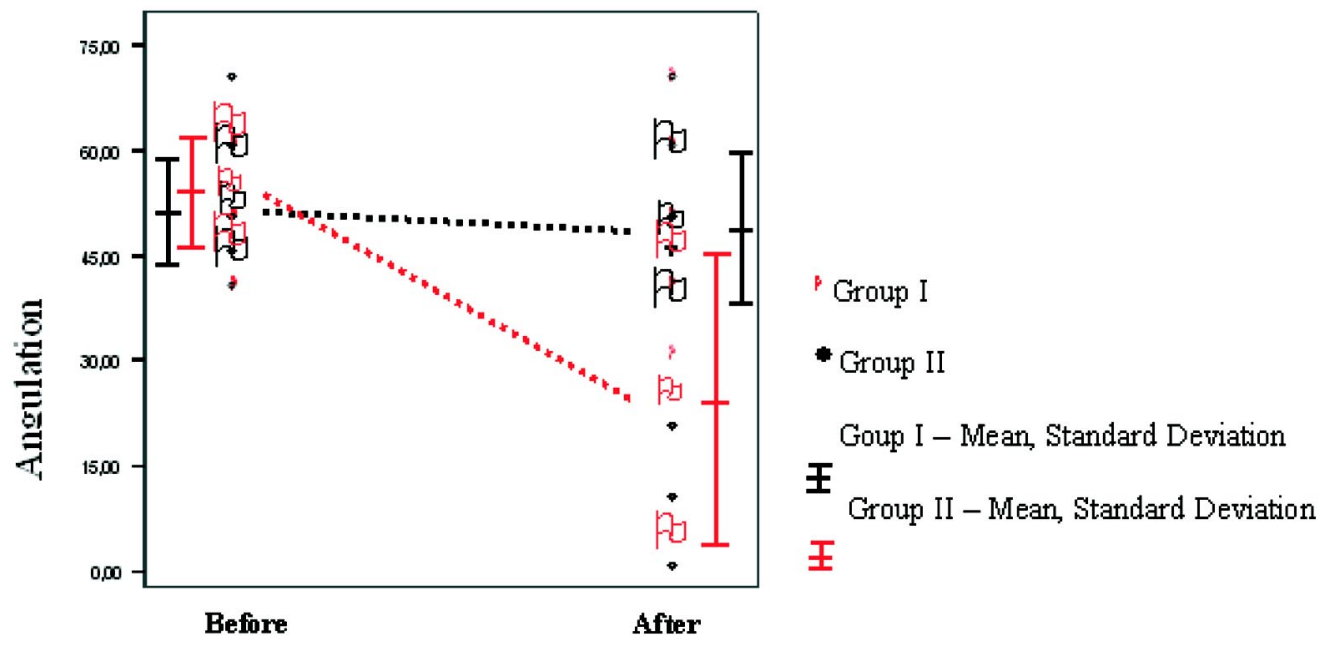

Figure 4 - Penile angulation (degrees) of patients from control (I) and study (II) groups (mean and standard deviation).

Table 1 - Diameter analysis by ultrasonography in the control (I) and study group (II) before and after treatment.

\begin{tabular}{llcc}
\hline Group & Diameter $(\mathbf{c m})$ & Mean & Standard Deviation \\
\hline I & Before & 1.08 & 0.26 \\
& After & 1.03 & 0.22 \\
II & Before & 1.32 & 0.61 \\
& After & 0.63 & 0.37 \\
\hline
\end{tabular}

Table 2 - Angulation (in degrees) assessed by photography taken with natural erection at home in the control (I) and study group (II) before and after treatment.

\begin{tabular}{lccc}
\hline Group & Angulation & Mean & Standard Deviation \\
\hline I & Before & 51.33 & 7.43 \\
& After & 48.67 & 10.6 \\
II & Before & 54.23 & 7.83 \\
& After & 24.42 & 20.8 \\
\hline
\end{tabular}




\section{DISCUSSION}

Peyronie's disease has been previously characterized as a process of gradual spontaneous resolution. However, only $14 \%$ of 97 patients followed during a 5 years period reported the disorders as resolving (11). The pain associated with erection commonly resolves with time but the angulation does not. Peyronie's disease affects the quality of life of both, the patient and his partner. Psychological effects due to Peyronie's disease are reported by the large majority of patients. The exact cause of the disease is unknown and most cases are probably idiopathic (8). Trauma or excessive bending to the erect penis may result in bleeding into the subtunical spaces making tunical delamination $(2,3)$. Therefore, trauma is thought to be the initiating factor in Peyronie's disease (11).

Whatever the cause might be, the outcome is the deposition of connective tissue into the tunica albuginea of the corpora cavernosa. There is initial perivascular inflammatory response that infiltrate the tunica albuginea, followed by the deposition of collagen and fibrin, leading to the penile plaques that cause the angulation deformity of the penis (14).

Although surgical correction of the angulation or the plaque is still considered the gold standard for treating Peyronie's disease, it certainly presents some important disadvantages. Some reduction in penile length and de novo impotence are not uncommon after surgery (10). As a result, minimally invasive treatments have been tried, including ortho-voltage radiation, ultrasound, short wave diathermy, laser therapy and shockwave lithotripsy. The exact mechanism of action of ESWT is still unknown (11) but there are 2 theories: a direct damage to the plaque (15) and an increased of vascular density of the area caused by ESWT, leading to an inflammatory reaction which results in lyses of the plaque and removal by macrophages (12).

In previous studies, the proportion of patients showing an improvement in their erection with ESWT therapy ranged from $47 \%$ to $71 \%(12,13)$. We associated vitamin $\mathrm{E}$, a common treatment for Peyronie's disease, using the dosage of $1.200 \mathrm{mg}$ daily (4 times greater than the dose proposal) (2) and ESWT in the attempt of improving the deformity in a non-invasive way. In this present study, 18 patients $(72 \%)$ referred improvement in the penile angulation. However, 8 patients referred improvement in the quality of their spontaneous erections. There were no major complications. Petechiae, skin hematoma, penile bruising and urethral bleeding were the only complications.

\section{CONCLUSION}

This initial experience with the association of high dose of vitamin E and ESWT is an effective and safe non-invasive treatment for Peyronie's disease. Further studies with long-term follow-up are recommended to confirm the efficacy of this therapy.

\section{REFERENCES}

1. Gelbard MK, Dorey F, James R: The natural history of Peyronie's disease. J Urol. 1990; 44: 1376-9.

2. Devine CJ Jr, Somers KD, Jordan GH, Schiossberg SM: Proposal: trauma as the cause of the Peyronie's lesion. J Urol. 1997; 157: 285-90.

3. Jarrow JP, Lowe FC: Penile trauma: an etiologic factor in Peyronie's disease and erectile dysfunction. J Urol. 1997; 158: 1388-90.

4. Wahl SM: Inflammation and growth factors. J Urol. 1997; 157: 303-5.

5. El-Sakka AI, Hassan MU, Nunes L, Bhatnagar RS, Yen TS, Lue TF: Histological and ultrastructural alterations in an animal model of Peyronie's disease. Br J Urol. 1998; 81: 445-452.

6. Smith BH: Subclinical Peyronie's disease. Am J Clin Pathol. 1969; 52: 385-90.

7. Gelbard MK, Dorsey F, James K: The natural history of Peyronie's disease. J Urol. 1990; 144: 1376-9.

8. Lindsay MB, Schain DM, Grambsch P, Benson RC, Beard CM, Kurland LT: The incidence of Peyronie's disease in Rochester, Minnesota, 1950 through 1984. J Urol. 1991; 146: 1007-9.

9. Carson CC, Jordan GH, Gelbard MK: Peyronie's disease: new concepts in etiology, diagnosis and treatment. Contemp Urol. 1999; 11: 44-64.

10. Schwarzer U, Sommer F, Klotz T, Braun M, Reifenrath B, Engelmann U: The prevalence of Peyronie's disease: results of a large survey. BJU Int. 2001, 88: 727-30.

11. Gholanni SS, Gonzalez-Cadavid NF, Lin CS, Rajfer J, Lue TF: Peyronie's disease: a review. J Urol. 2003; 169: 1234-41. 
12. Husain J, Lynn MN, Jones DK, Collins GN, O'Reilly PH: Extra corporeal shockwave therapy in the management of Peyronie's disease: initial experience. BJU Int. 2000; 86: 466-8.

13. Hamm R, McLarty E, Ashdown J, Natale S, Dicthison A: Peyronie's disease: the Plymonth experience of extracorporeal shockwave treatment. BJU Int. 2001, 87: 849-52, 2001.

\section{Correspondence address:}

Dr. Joaquim de Almeida Claro

R. Brig. Faria Lima, 1713, conj. 72

São Paulo, SP, 01452-001, Brazil

Fax: + $55113031-6444$

E-mail: joaquimclaro@hotmail.com

\section{EDITORIAL COMMENT}

The authors present a further study on the use of extracorporeal shock wave therapy (ESWT) for the treatment of Peyronie's disease. In contrast to the most recently published series describing disappointing results under prospective long-term designs $(1,2)$ the authors present ESWT as successful method for treating penile curvatures. These results are also contradictory to the recently published exploratory metaanalysis of the studies published so far (3). In this meta-analysis no clear effect of ESWT on penile curvature and plaque size was evident (3)

How could these remarkably differences be explained? Unfortunately the authors do not discuss these aspects. In contrast to the previous published in the literature the authors combined classical ESWT with the oral administration of high dosage of vitamin E, four times higher than normal. Could this aspect be the key aspect to improve the effect of ESWT? Regarding to the published literature the use of vitamin E does not seem effective compared to the natural history $(4,5)$. Thus it does not seem very reasonable to combine to ineffective methods for treatment of Peyronie's disease that is really a disorder difficult to cure. However, the data of the present study seem to be impressive because the only administration of vitamin $\mathrm{E}$ as control group is not effective - as previously described.
14. Somers KD, Dawson DM: Fibrin deposition in Peyronie's disease plaque. J Urol. 1997; 157: 311-5.

15. Lingeman JE, McAteer JA, Kempon SA, Evan AP: Bioeffects of extracorporeal shock wave lithotripsy: strategy for research and treatment. Urol Clin North Amer.1988; 15: 507-14.

Received: April 30, 2004 Accepted after revision: May 20, 2004
Although ESWT is not favored as standard treatment by our Peyronie's study group $(2,3)$ the authors should be encouraged for further studies of their concept.

\section{References}

1. Strobe RT, Sutter S, Sutter T, Hairy D: Extracorporeal shockwave therapy for Peyronie's disease does nor corrects penile deformity. Int J Impotence Res. 2004 (Epub ahead of print).

2. Hauck EW, Hauptmann A, Bschleipfer T, Schmelz HU, Altinkilic BM, Weidner W: Questionable efficacy of extracorporeal shock wave therapy in Peyronie's: results of a prospective approach. J Urol. 2004, 171: 269-299.

3. Hauck EW, Mueller UO, Bschleipfer T, Schmetz HU, Diemer T, Weidner W: Extracorporeal shock wave therapy for Peyronie's disease: exploratory meta-analysis of clinical trials. J Urol. 2004; 171: 740-745.

4. Gelbard NK, Dorey F, James K: The natural history of Peyronie's disease. J Urol. 1990; 144: 1376-1379.

5. Pryor JP, Farell CR: Controlled clinical trial of vitamin E in Peyronie's disease. Prog Reprod Biol Med. 1983; 9: 41-45.

Dr. Wolfgang Weidner Director, Department of Urology Justus Liebig University Giessen Giessen, Germany 